time/hours, and 4 physico-chemical exposures. Weighted robust Poisson regression models were used to investigate the associations between occupational exposures in 2013 and incident poor well-being in 2016 after adjustment for covariates, in men and women separately.

Results The incidence rate of poor well-being in 2016 was $10.3 \%$ among men and $16.8 \%$ among women. Significant prospective associations were found between a wide range of PWFs and poor well-being, and the risk increased with the number of these factors globally and within almost all psychosocial dimensions (except work demands in men and workplace violence in both genders). Among the other studied occupational exposures, noise was prospectively associated with poor well-being in women.

Conclusion Working conditions, especially PWFs, could have a negative impact on well-being and exposure to multiple PWFs could increase the risk of poor well-being still further.

\section{P-53 MULTIPLE EXPOSURES TO OCCUPATIONAL FACTORS AND SLEEP PROBLEMS AMONG EMPLOYEES IN FRANCE}

'Sandrine Bertrais, Noëmie André, Jean-François Chastang, Isabelle Niedhammer, Marilyne Bèque. 'Inserm DR GRAND OUEST, France

\subsection{6/OEM-2021-EPI.179}

Objective Many studies suggest that working conditions may have an impact on health, including sleep. One of the main limitations inthe literature, however, is that studies have explored a limited number of occupational exposures and have not addressed the issue of multiple exposures. Our objective was to study the associations of a large variety of occupational exposures with sleep problems, and to assess the impact of multiple occupational exposures on this outcome.

Methods The study sample consisted of 20430 employees aged 15-65 years (8579 men, 11851 women) included in the representative sample of the working population from the 2016 French national survey on working conditions. Participants were classified as having sleep problems if they reported sleep disturbances and/or sleep medication, at least several times a week. Occupational exposures were: 21 psychosocial work factors (PWFs) further grouped in five dimensions (work demands, work organization and job content, interpersonal relations and leadership, work-individual interface, workplace violence), four working time/hours factors and four physicochemical exposures. Weighted robust Poisson regressions were performed to study the associations between occupational exposures and sleep problems after adjustment for covariates, in men and women separately.

Results Among the studied working time/hours factors, only night work among women was associated with sleep problems, while most of psychosocial work factors and physico-chemical exposures were significantly associated with sleep problems. Stronger exposure-outcome associations were found in men for some PWFs. The odds of sleep problems increased with increasing number of exposures for most dimensions of PWFs, and with increasing number of physico-chemical exposures (non-significant trend in women).

Conclusion Psychosocial and physico-chemical exposures were found to be associated with sleep problems, and the higher the number of exposures, the higher the odds of sleep problems. More studies are needed on multiple occupational exposures in association with sleep problems among working populations.

\section{P-54 MULTIPLE EXPOSURE TO PSYCHOSOCIAL FACTORS AT WORK AND OTHER OCCUPATIONAL FACTORS AND COMMON MENTAL DISORDERS AMONG EMPLOYEES IN FRANCE}

${ }^{1}$ Sandrine Bertrais, Jean-François Chastang, Isabelle Niedhammer, Amélie Mauroux. ${ }^{1} / n s e r m$ DR GRAND OUEST, France

\subsection{6/OEM-2021-EPI.180}

Objective Knowledge on the cumulative effects of psychosocial work factors on mental health is sparse, and other occupational exposures such as those from the physical work environment remain understudied in this topic. Therefore, this study aimed to examine the associations between various types of occupational factors, including a large variety of psychosocial work factors (PWFs), and major depressive episode (MDE) and generalized anxiety disorder (GAD) in the French working population.

Methods The study was based on data from the 2016 national survey on working conditions conducted on a representative sample of workers, including 20430 employees aged 15-65 years (8579 men, 11851 women). The MINI standardized diagnostic interview was used to assess MDE and GAD. The following occupational exposures were studied: 21 PWFs grouped in five dimensions (work demands, work organization and job content, interpersonal relations and leadership, workindividual interface, workplace violence), four working time/ hours factors and four physico-chemical exposures. The associations between occupational exposures and MDE/GAD were assessed in each gender separately using weighted logistic regression models.

Results We found a higher prevalence of MDE and GAD among women than among men $(8.6 \%$ and $8.7 \%$ respectively versus $4.3 \%$ and $4.6 \%$ ). There were significant associations between most psychosocial work factors, andMDE and/or GAD. The odds of MDE/GAD increased with the number of exposures for all psychosocial dimensions, except workplace violence. The odds of MDE also increased with multiple physico-chemical exposures. No association was found between working time/hours factors and MDE/GAD, except between unsocial work days, shift work and MDE in women.

Conclusion Our results highlighted the negative impact of being exposed to multiple PWFs for depression and anxiety, and showed a cumulative association of the four studied physico-chemical exposures with depression. More research is needed on the effect of the accumulation of occupational exposures on these outcomes in working populations.

\section{P-60 RELATIONS BETWEEN WORK-RELATED FACTORS AND STRESS AND INJURY AMONG JANITORS}

${ }^{1}$ Susan Gerberich, Andrew Ryan, Adam Schwartz, Deirdre Green, Patricia McGovern, Hyun Kim, Rony Arauz. 'University of Minnesota, United States

\subsection{6/OEM-2021-EPI.181}

Introduction While a body of literature identifies relations between injury occurrence and resulting stress, literature 
relevant to relations between work factors and stress and injury occurrence is limited.

Objectives To identify the potential relations between: 1) work-related factors and stress and 2) stress and depression and injury outcomes in a high risk, understudied population.

Methods Specially designed questionnaires were disseminated to 1,200 full-time unionized janitors to collect information on their injury occurrences, personal characteristics, health history, and work-related exposures, for two sequential six-month periods. Risk ratios (RRs) and 95\% confidence intervals (CIs) were calculated using multivariable Poisson regression with robust error variances, and included bias adjustment for nonresponse and adjustment for within-person correlation using general estimating equations (GEEs).

Results 527 total observations among 390 janitors identified associations between the following exposures and high/low stress outcome (collapsed 5 point Likert scale - often/very much versus (vs) not at all/very little/sometimes): work environment factors (range $=1$, terrible/unhappy/mostly dissatisfied; 2, mixed feelings; 3, mostly satisfied/pleased delighted) how they felt about their job (1 vs 3 - RR 4.50; CI 2.38, 8.52); where they worked ( 1 vs 3 - RR 3.46; CI 2.03, 5.92); resources available for their job (1 vs 3 - RR 1.77 CI 1.14, 2.76); and job mentally or physically demanding (high/very high vs very low/low/medium demand) RR 2.49; CI 1.26, 4.93 and RR 3.74; CI 1.37, 10.25, respectively. High vs low stress exposure was associated with outcomes: diagnosed depression yes/no (RR 4.79; CI 2.22, 10.36); and risk of injury (RR 1.45; CI 1.00, 2.10).

Conclusions This analysis enabled identification of: workrelated factors associated with reported stress; the relation between stress and depression; and risk of injury among those reporting stress levels. These findings serve as a basis for future research and relevant interventions to facilitate optimal working environments.

\section{P-62 SICKNESS ABSENCE AND PSYCHOSOCIAL CONSTRAINTS AT WORK: A CROSS-SECTIONAL STUDY AT A UNIVERSITY HOSPITAL IN TUNISIA}

${ }^{1}$ Aouatef Mahfoudh, Nesrine Mars, Noura Bel Haj, Ines Rassas, Amira Omrane, Taoufik Khalfallah. 'University of Monastir, Tunisia

\subsection{6/OEM-2021-EPI.182}

Background The relationship between work and health is complex and bidirectional. It has individual and collective aspects resulting from working conditions and interpersonal relationships. Absenteeism, a complex phenomenon, represents for some workers the expression of health and work relationship. It is considered as an indicator of a person's physical and mental health status. Aims: To identify determinants of sickness absence among nurses in a Tunisian university hospital.

Methods A cross-sectional study carried out in a Tunisian University Hospital. We included medical absences declared to the employer by nurses during one year. The study was conducted in two successive phases: data collection of absence from the hospital direction (human resources), then a standardized self-administered questionnaire was distributed to the target population. This questionnaire included a socioprofessional survey and a questionnaire on Psychological and
Organizational Constraints (POC), using a version applicable to nurses (IDE).

Results The global absence rate was 6.58. Absenteeism was statistically associated with age $(p=0.035)$, marital status ( $p$ $=0.005)$ and the presence of medical and surgical history $(\mathrm{p}$ $\left.<10^{-3}\right)$. The number of absence days was statistically higher among nurses with a pathological professional history $(\mathrm{p}=$ 0.019 ) and among those with an occupational tenure higher than 15 years $(\mathrm{p}=0.034)$. For the psycho-organizational constraints studied, the period of absence was statistically associated with the organization that did not allow communication $(p=0.001)$, the lack of support from the health manager $(p$ $=0.001)$ and a shortage of staff $\left(\mathrm{p}<10^{-3}\right)$. The multivariate regression analysis showed as determinants of absenteeism: medical history $\left(\mathrm{p}<10^{-3} ; 95 \% \mathrm{CI}=[2.21 ; 8.18]\right)$, surgical history $(p=0.001 ; 95 \% \mathrm{CI}=[1.71 ; 9.12])$ and the lack of support from the health manager $(\mathrm{p}=0.001 ; 95 \%$ CI $=$ $[1.58 ; 6.34])$.

Conclusion This study allowed us to identify some determinants of absenteeism among nurses.

\section{P-63 SOCIO-ECONOMIC ISSUES OF OCCUPATIONAL MUSCULOSKELETAL DISORDERS}

${ }^{1}$ Aouatef Mahfoudh, Olfa Jlassi, Amira Omrane, Noura Bel Haj, Asma Kheder, Ines Rassas, Taoufik Khalfallah. 'University of Monastir, Tunisia

\subsection{6/OEM-2021-EPI.183}

Aims To assess determinants of absenteeism among employees with occupational musculoskeletal disorders (MSDs).

Methods A descriptive cross-sectional study carried out at the Department of Occupational Medicine in the University Hospital of Tunisia. The survey took place during the year 2019 and was conducted using a pre-established questionnaire, relating to the description of socio-demographic data and the assessment of work capacity by the Work Ability Index questionnaire 'WAI'. The study of absenteeism linked to MSDs was carried out using the Work Performance Questionnaire 'WPQ'.

Result A total of 63 participants, all females, mean age $41 \pm$ 8 years, had consulted for a musculoskeletal problem. In this series, $58 \%$ of these women had a low level of education (primary). They were married in $77 \%$ of cases with more than two children in charge in $61 \%$ of cases. The average professional length was $20 \pm 7$ years. Sectors of activity were clothing (92\%), food processing (4\%), and the automotive wiring industry (4\%). The capacity for work perceived by all the operators was low in $85 \%$ of cases and average in $15 \%$ of the cases. The mean relative absenteeism of the study population was $31 \%$. Absenteeism was significantly correlated: with age $(\mathrm{p}=0.03)$, with the work of the spouse $(\mathrm{p}=$ 0.006), with the distance between the place of work and the residence $(p=0.015)$, with the sector $(p=0.001)$ and professional seniority $(p=0.032)$. In this work, the explanatory model of medical absenteeism linked to work-related MSDs was statically linked to professional length of service ( $p$ $=0.02 ;$ OR $=2.3, \mathrm{IC}=[1.3-4.5])$.

Conclusion Musculoskeletal disorders have various repercussions in terms of suffering and interruption of professional careers of employees, but also high economic costs. As a result, a comprehensive and integrated prevention approach must be put in place. 